\title{
Entre la evasión y la nostalgia. Estrategias de la neoruralidad desde la economía social
}

\author{
Between avoidance and nostalgia. Neorural strategies in the context of the Social Economy
}

\author{
Óscar Fernández Álvarez \\ Universidad de León, Campus de Vegazana. León (España) \\ oscar.fernandez@unileon.es
}

\author{
NUEVAS RURALIDADES \\ MONOGRÁFICO COORDINADO POR SHARON R. ROSEMAN, SANTIAGO PRADO Y XERARDO PEREIRO
}

\begin{abstract}
RESUMEN
Este trabajo tiene como objeto analizar la estrategia desarrollada para la integración en el sistema económico y social de familias neo-rurales a través de una sociedad agraria de transformación y del movimiento "wwoofers" o red internacional de voluntariado de granjas orgánicas. Después de una breve revisión teórica y conceptual que nos permite incorporar conceptos para nuestro análisis, mostramos la experiencia propiamente, aspecto central de este trabajo, y que se desarrolla en dos pueblos de la pedanía de Brazuelo, próxima a la localidad de Astorga, en la provincia de León. Estudiamos los aspectos técnicos de los dos elementos que han permitido el mantenimiento y reproducción de la experiencia, las sociedades agrarias de transformación y los "wwoofers". La metodología usada en la investigación se basa en la observación, en entrevistas en profundidad y la realización de grupos de discusión. El análisis de los datos muestra que, aunque no es un proceso importante cuantitativamente, como está documentado en otros estudios de caso, se producen cambios significativos en la estructura económica y social. La iniciativa desarrollada a través de una configuración de economía social, no buscar tanto conseguir unos beneficios económicos, que también, sino compartir el aprendizaje y transmisión de un modo de vida ecológico a través de las granjas de productos orgánicos.
\end{abstract}

This study analyses the strategy developed by neorural families for their integration into the economic and social system through an agrarian society of transformation and the "wwoofers" movement, an international network of volunteers working on organic farms. After a brief theoretical and conceptual review introducing the concepts used in our analysis, we describe the experience itself -a central aspect of this work- as it unfolds in two villages of the District of Brazuelo, near the town of Astorga, in the province of León. We discuss organizational and other aspects of the two social elements that have allowed the maintenance and reproduction of the experience, the agrarian societies of transformation and the "wwoofers". The methodology used in the research is based on observation, in-depth interviews and a series of discussion groups. The analysis of the data shows that, although it is not an important process quantitatively, as documented in other case studies, this way of farming leads to significant changes in the economic and social structure. The initiative, developed through a social-economy configuration, seeks not only economic benefits, but also to share what it is to learn about and transmit an organic lifestyle through organic farms.
\end{abstract}

PALABRAS CLAVE

neo-ruralidad | SAT (Sociedad Agraria de Transformación) | WWOOF Economía social. León KEYWORDS

neo-rurality | AST (Agrarian Society of Transformation) | WWOOF Social economy. León

\section{Introducción (1)}

El campo constituye sin duda, un entorno cada vez más buscado por urbanitas, y este fenómeno está promoviendo una serie de transformaciones. Además, los cambios en el modelo socioeconómico que se vienen produciendo desde las dos últimas décadas del siglo XX afectan también al medio rural. Por ejemplo, los diferentes intentos que se vienen promoviendo por parte de las administraciones para fijar población en zonas rurales, en las que en otra época, principalmente en la década de 1960, incitaron a 
su abandono en nombre del desarrollismo; o el caso de los grupos alternativos que se instalan en el campo a partir de la década de 1980, en busca de un paraíso perdido y añorado en su vida en la ciudad, ilustran esta cuestión.

Son éstos, dos procesos que hemos observando en el trabajo de campo que venimos desarrollando para el análisis de la neoruralidad en la provincia de León.

Dos aspectos que, en el estudio llevado a cabo, uno conduce principalmente a la geriatrización del espacio rural, el segundo, principalmente a la gentrificación de ese espacio. No son excluyentes pues al menos el segundo, a veces, puede incorporar al primero. Aquí no vamos a centrar en el segundo aspecto: nuevos grupos y familias que se asientan en el ámbito rural en busca de una forma de vida diferente, buscando una relación más cercana con la naturaleza.

Comenzaremos por una breve revisión teórica y conceptual sobre el tema que nos permite incorporar conceptos para nuestro análisis. A continuación mostramos la experiencia de neo-ruralidad en la que basamos este trabajo, y que se desarrolla en dos pueblos de la pedanía de Brazuelo, León. Después, estudiamos dos aspectos que han permitido el mantenimiento en el tiempo de la experiencia, y que son las sociedades agrarias de transformación y los wwoofers, o voluntariado de granjas orgánicas. Y por ultimo planteamos una discusión sobre los datos aportados y las conclusiones.

\section{Neoruralidad como marco teórico}

La ruralidad es considerada por un número cada vez más amplio de autores, como una alternativa a la crisis de la vida en la ciudad, como un ámbito donde se presentan innumerables oportunidades para aquellos que buscan otro modo de vida. Pero esta búsqueda por parte de los urbanitas de un modo de vida en el campo, el deseo de vida en microsociedades y en plena naturaleza, no es reciente. El naturalismo convencional enraíza en las mentalidades del XIX y principios del XX, y posteriormente, la creciente desagrarización del campo, las mejoras económicas, en los sistemas de comunicación, en las formas de trabajo con las nuevas tecnologías, que permiten el trabajo a distancia, la nueva concepción urbana de lo rural, han puesto en cuestión la necesidad de habitar la ciudad y nos ayuda a explicar el creciente aumento que se está produciendo en el proceso de vuelta al campo. Quizá, en esta evolución, la década de los setenta reveló las premisas de ese entusiasmo por el campo y, a pesar de que ese neoruralismo ha sido descrito por muchos como un movimiento utópico, las iniciativas que se han ido implantando en materia de desarrollo rural son dignas de tener en cuenta.

En una rápida revisión teórica de las aportaciones que se han hecho en el tema de las nuevas ruralidades es necesario destacar el trabajo de síntesis de Delgado (1999), que agrupa la literatura en tres bloques temáticos: uno, globalización y neoliberalismo; dos, flexibilización y agrosistemas, y tres, patrones espaciales. En el primer bloque se cita a Long (1996), quien desde la interrelación que encuentra en la articulación de lo global y lo local, la disolución del antiguo Estado-nación y la emergencia de nuevos movimientos sociales, percibe la globalización como un "proceso socialmente construido y constantemente renegociado". Así frente al planteamiento de centro-periferia, propone un ordenamiento en términos de homogeneización-diversidad que le lleva a formular los conceptos de propiamente "nueva ruralidad", "localización" y "relocalización". En el segundo bloque, flexibilización y agrosistemas, frente al "planteamiento anacrónico rural-urbano", se retoman sugestivos conceptos como rurbanización, agrociudades y agricultura periurbana. Se cita a Lara (1996), quien apunta que los movimientos actuales producen tanto una "urbanización de la vida campesina, como una ruralización de la periferia urbana". En el tercer bloque, patrones espaciales de la nueva ruralidad, lo más significativo, quizá, es que se da cuenta de la obsolescencia de las definiciones de la ruralidad contenida en el discurso de organismos internacionales comola UEola OCDE, que tutelan políticas de repoblación del campo, por lo que sería necesario una nueva revisión de sus criterios.

Desde otra perspectiva de la antropología y geografía rural, y con un planteamiento transnacional, comparando tres estudios de caso de Estados Unidos, Francia y España, Nates Cruz y Raymond (2007) 
analizan las emigraciones al medio rural bajo tres perspectivas de "retorno": a la naturaleza, en busca de un modo de vida austero y en armonía con la naturaleza; al campo, en un modo de vida urbano que goza de las bondades del campo, y a la tierra, como modo de vida neo-campesina con equilibrio medioambiental. Además, tratan de estudiar el rol de esos flujos migratorios en las recomposiciones territoriales, sociales, económicas, culturales y políticas de los lugares de acogida, así como cuáles son las percepciones de los habitantes de estos lugares de acogida.

Por otra parte, el trabajo de Salas, Rivemar y Velasco (2011), aunque centrado en el caso de México, aporta nuevas herramientas analíticas y metodológicas para entender las nuevas ruralidades y los procesos de cambio que están sucediendo en el campo, que bien pueden ser aplicadas a nuestro país. De la misma forma, y desde una perspectiva multidireccional y para el caso ibérico, la síntesis de estudios de caso que se recoge en Pereiro, Prado Conde y Roseman (2011), muestra que la versatilidad temática, la variabilidad geográfica y la construcción teórica expuesta, son ejemplo de la riqueza analítica que está adquiriendo el tema en cuestión.

La revisión de la literatura sobre el tema, nos lleva a plantear que, desde un contexto de urbanización creciente, hay una búsqueda insistente por instalarse en las zonas rurales, donde lo rural en si mismo ya no constituye un objeto de investigación, sino un sector más de la sociedad. En ese sector de investigación pueden ser planteados y estudiadas toda una serie de cuestiones que afectan tanto, de forma particular al hombre y su relación con el entorno, con la naturaleza, como a otra serie de manifestaciones sociales y culturales, y afectan, y ya están siendo contempladas, ala Historia, Geografía, Antropología y otras ciencias sociales y humanas. Lo rural se convierte en un espacio integrado, globalizado, y por tanto, ya no parece pertinente hablar de lo rural haciendo referencia a su pasado, sobre todo, como dimensión social, es decir, con relación a lo urbano. Lo rural empieza a tomar nueva forma de poder ser, a tener otro significado, un significado que nunca antes había tenido.

El trabajo de campo que se muestra aquí hace referencia al desarrollado en Requejo de Pradorrey y Bonillos, dos pueblos de la pedanía de Brazuelo, próximos a la localidad de Astorga y a50 kilómetros de León. Cuentan con una población que roza el medio centenar de habitantes.

La metodología usada en la investigación se basó en la observación, en entrevistas en profundidad que, de modo sui géneris por el proceso cómo se desarrollo el trabajo de campo, bien pueden ser considerados como grupos de discusión, pues es la técnica que mejor que se nos revelado para descubrir el discurso argumental que se elabora en torno a esta situación por diversas partes.

\section{Neo-rurales en Brazuelo}

El concepto de movimiento neo-rural, dice Folch Monclús (2011), surge para designar a aquellos grupos de personas que por decisión propia abandonan su lugar de origen, generalmente la ciudad, para establecerse en un entorno rural con la intención de alejarse de todo aquello que suponen los valores de la vida urbana, en su sentido más peyorativo, desde el punto de vista económico y social, y para establecer un proyecto de vida comunitario en el medio natural. Por la estética e ideología de estos grupos se les identificó con movimientos sociales contraculturales o alternativos. En cierto modo, eran herederos de los movimientos libertarios aparecidos desde de finales del siglo XIX y más propiamente en el $X X$, que con una visión heterodoxa, incorporaban planteamientos utópicos, igualitarios, anticapitaslistas o ecológicos.

Este movimiento, en ese aspecto contestatario, se integra en un proceso más amplio que el propiamente neo-rural. Tiene una dinámica marcadamente global que surge, como decíamos al principio, del avance de las nuevas tecnologías, de la dasagrarización del campo, de cuestionarse la vida en las ciudades y de un intento de experimentar la vida en comunidad. De cualquier manera, y para nuestro ámbito, tiene su precedente, de forma general, en un pasado compartido por gran parte del campo español de fuerte despoblación, pérdida de peso demográfico, económico y político y, de forma específica para nuestro caso, en la aparición de unas primeras experiencias neorrurales de la década de 1980, con gran 
continuidad en años posteriores, hasta el punto de revivir pueblos que habían sido abandonados. El caso más significativo, y en su día famoso, por la repercusión a nivel demográfico, y de dinamismo económico y social, es el de Matavenero, en el Bierzo leonés, del que proceden algunos de los pobladores actuales de Requejo de Pradorrey, nuestra zona de estudio.

En aquél caso, el retorno al campo adquirió una fuerte carga simbólica como lugar para la construcción de la sociedad ideal y de reencuentro con la naturaleza, a modo de "paraíso perdido", se convirtió en el espacio ideal para su realización. Sólo tenían que seguir y actualizar las pautas de comportamiento preexistentes durante siglos y que habían sido perdidas con el abandono de los pueblos por los autóctonos, pero no olvidadas, pues de su existencia nos habla la documentación de los historiadores. En este sentido, un claro exponente para la provincia de León fueron los trabajos de Rubio Pérez (1993). El proceso seguido a continuación es similar al que describe, por ejemplo, Folch Monclús (2011) para el caso dela Terreta, en el Pirineo de Lleida: una agricultura colectivizada, y de perfil tradicional, con explotación comunitaria de recursos naturales, "que junto a la influencia de las nuevas sensibilidades ecológicas, descubrieron las ventajas de las formas de vida tradicionales, convirtiéndolas en alternativa, e identificando al campesino con una especie en extinción que había que preservar y emular".

Actualmente, estos grupos "alternativos" llegan al campo, con la idea de conseguir una calidad de vida, un gran espacio de naturaleza, y construir ahí un entorno donde encontrar, de nuevo, el "paraíso perdido". Este es el caso de nuestros informantes de la pedanía de Brazuelo: Familias de neorrurales o "ciudadanos alternativos" como prefieren denominarse, sin otra conexión que el destino, han restablecido su vida, hace más de dos años, en dos pueblos de la Maragatería más pobre, separados por diez kilómetros: Bonillos y Requejo de Pradorrey. "No somos una comunidad al estilo tradicional". Tampoco encajan estrictamente en el perfil de eco-aldea. "Somos un grupo de familias e individuos de aquí y de allá". Su procedencia es tan dispar como Alemania, Brasil o Madrid. "Y aquí se puede participar de las labores de una granja orgánica a cambio de manutención y alojamiento para ser autosuficientes".

Vamos a tomar el ejemplo de una de las familias establecidas en Bonillos. El planteamiento de partida es contundente: se establecieron en este pueblo "cansados de la vida de Madrid". Él, padre de familia, trabaja ahora en León y se desplaza para hacer los turnos que tiene asignados en su empresa. Piensa que el tiempo que empleaba en Madrid para desplazarse de su casa al trabajo, comparado con estos 50 kilómetrosde ahora, no es nada. Y lo más importante: "el entorno que encuentras cuando llegas a casa, no se puede comparar, no son unas paredes de pladur". El planteamiento de llegada es concluyente: Ia gran ciudad como cúmulo de diversos males. La polución, el ruido, la inseguridad, pérdida de tiempo, cada vez son más difíciles de soportar, "puedes llamarlo nivel de vida pero no calidad de vida", nos dice. Frente a estos males, en el imaginario se opone el campo, al que se le atribuyen caracteres antitéticos: equilibrio con la naturaleza y con uno mismo, vida social en convivencia, etc.

Sus dos hijos estudian en Astorga y "aquí son felices". El hecho de que se tengan que desplazar para ir a la escuela no es visto como un problema: "Cuando vuelven a media tarde, el entrono les compensa, así como el poder correr y jugar, salir cerca de la casa sin la amenaza del asfalto". Para esta pareja, tener hijos contribuye a la integración y la adaptación de cualquier nuevo residente. Cuando hay niños en el medio rural, donde generalmente la media de edad es elevada y el despoblamiento es una seria amenaza, significa estímulo y un motivo de esperanza para todo el pueblo que, lógicamente, recibe con los brazos abiertos a los nuevos vecinos. Además, suele ser un signo de que se está instalado de forma definitiva y de que ya se tiene cierto arraigo en el lugar. No hace falta hablar de cómo los niños fomentan la socialización e interacción de los adultos.

Ella es profesional de la arquitectura y puede realizar su trabajo desde casa, en el pueblo. Como parte de su profesión, ha rehabilitado una antigua casa de arriero maragato, donde viven ahora, y una parte la dedican a casa rural. De alguna forma, esta madre de familia, pertenece a ese sector de profesionales que, amparados en las nuevas tecnologías, hacen del pueblo su lugar de residencia y trabajo. Este es un elemento que de forma bastante recurrente ha mostrado la literatura sobre el tema: más allá de la llegada de nuevos pobladores, se muestra una gentrificación de territorios rurales. Y a pesar de la reticencia a su planteamiento en un ámbito no urbano (Smith 1996), no se puede negar la difusión que está teniendo en 
la literatura sobre el medio rural (rural gentrification), como muestra el debate y disquisiciones que exponen ampliamente Nates Cruz y Raymond (2007: 47-55). Este proceso, impulsado por sectores sociales académicamente preparados o profesionales con un poder adquisitivo y medio alto, busca instalarse en el campo y crear un modo de vida distinto al que se impone en el sistema social y económico hegemónico. Es significativo también, observar, cómo aquí, ahora el campo representa los valores de vida en libertad que en otro tiempo tuvo la ciudad. Esto nos permite comprobar que, lo que en otro tiempo se llamaban migraciones alternativas, contestataria o utópicas, lejos de desaparecer, siguen expresándose bajo otras formas. El campo, en términos de atracción, sigue suscitando ilusiones en el imaginario social, es decir, la ruralidad deseada por una población urbana con "mal de campo" según denominaba De Ravignan (1996) o déficit o "deseo de campo".

Su casa rural que habitan y regentan se llama "La Casadel Arzipreste". Pertenece a una sociedad agraria de transformación de la que hablaremos más adelante. De hecho, hay más personas vinculadas a este proyecto para aprender, ayudar, etc., por ejemplo, otro arquitecto, que procede y trabajó y vivió siempre en Madrid y ahora realiza su trabajo desde el pueblo gracias a internet; otra chica, que llegó de Brasil y también colabora en la Casa. En Bonillos conviven con otros pocos vecinos del lugar, truly rural (Harper 1987).

En Requejo de Prodorrey, destaca una casa imponente del siglo XVIII que se conoce también como "La Casadel Arzipreste". Los factores históricos y culturales evocados en los discursos permiten explicar la relativa debilidad del control ejercido por los nativos frente a los llegados de fuera. Éstos han ido llegando desde hace tres años, han rehabilitado algunas casas dando valor al patrimonio local y a los símbolos del pasado de los cuales, los nativos no pueden ocuparse o no han querido. Así, los modos ancestrales de la cultura agrícola del lugar son reutilizados por los neo-rurales, pues son ellos lo que pueden rehabilitar las casas maragatas de los siglos XVII y XVIII. El espíritu de "La Casa" de Requejo, y ya también del pueblo entero, es Claudia. Alemana de origen, tiene antecedentes familiares en Matavenero, el caso mencionado de la comarca del Bierzo. Fue una experiencia pionera de neo-ruralidad, bajo un movimiento de tipo rainbow, que tuvo gran repercusión mediática a partir de 1980 y fue objeto de estudio en la obra de Nates Cruz y Raymond (2007). Hobo, su padre, está aquí ahora. Vivió en Matavenero durante diez años, por lo que ella "hasta podría ser considerada segunda generación de neo-rurales de la familia". Tiene también un taller de artesanía. De objetos que después vende en ferias o mercados de la comarca. La familia de Claudia fue una de los primeras pobladores de tipis o viviendas de nómadas que se establecieron en ese pueblo. Ahora, además de mantener en pie la casa y corrales que ocupa, promueve una iniciativa peculiar e insólita, incorporándose a la red wwoofers, o de voluntariado de granjas orgánicas, de la que también hablaremos más adelante.

Es significativo que la comunidad de Bonillos ha establecido una conexión muy fluida con el pueblo vecino de Requejo de Pradorrey, y entre los grupos de ambos pueblos han establecido esta sociedad agraria de transformación. "La Casadel Arzipreste" ha servido como elemento aglutinador de desarrollo y mantenimiento de este estilo de vida. Es una sociedad agraria de transformación (SAT), y además está integrada en el movimiento wwoof, una red internacional de intercambio en materia de vida sostenible. Veamos estos elementos en detalle.

\section{SAT como elemento de desarrollo rural, o ¿cómo actúa la economía social?}

Dice Vargas Vasserot (2010) que una sociedad agraria de transformación (SAT) es una figura híbrida entre las sociedades civiles, cooperativas agrarias y sociedades mercantiles. Son sociedades específicamente participativas y esencialmente mutualistas. Su origen, parece ser que se encuentra en Ios Ilamados Grupos Sindicales de Colonización (GSC), formas asociativas creadas trasla Guerra Civil española, a modo de entes intermedios entre agricultores y el Estado, con ciertos vínculos semipúblicos y un marcado carácter consorcial. La SAT, es una fórmula de asociacionismo agrario peculiar del ordenamiento jurídico español que ha sido de gran utilidad para el desarrollo de determinada zonas, incluidas las de ruralidad profunda (Di Méo 1998) o como la que nos ocupa, de "La Casadel Arzipreste". 
Una sociedad de este tipo les permite tener acceso a una agilidad de constitución, de gestión o ventajas económicas por parte de la administración, etc.

Quizá uno pueda pensar que el origen del término "transformación" está en que estas sociedades tenían como finalidad la transformación de los productos entregados por los socios. Pero no, no es así, pues con esta forma asociativa se pretendía "transformar el panorama agrario español". Y, en cierto modo, así fue. Tal como muestra Roman Cervantes (2008), durante años, sobre todo hasta que se procedió a una mejor regulación del régimen legal de las cooperativas agrarias a finales de los ochenta, fue el referente del asociacionismo agrario en España, y así sigue siendo en algunas zonas indicadas. Sin entrar demasiado en cuestiones técnica sobre su regulación, es necesario conocer algunos detalles para entender su funcionamiento. La razón de la vigencia de las SAT y de las peculiares normativas permisivas que la regulan, hay que buscarla en la gran flexibilidad del tipo social y la no imposición de obligaciones económicas y financieras severas. También, en el escaso control tanto notarial como registral al que están sometidas estas sociedades, pues únicamente se inscriben en un registro administrativo dependiente del Ministerio de Agricultura y Medio Ambiente, o del organismo autonómico correspondiente. Todo ello ha favorecido su proliferación en el sector agrario. De este modo, a pesar de disfrutar de un trato fiscal peor que el de las cooperativas agrarias, muchos operadores económicos prefieren constituirse en empresas de este tipo, cómodas y flexibles, antes que en las complicadas y farragosas cooperativas. A pesar de la unánime crítica al estatuto legal de las SAT, que se puede entrever en la literatura sobre el tema, la teórica bonanza de esta forma de asociacionismo agrario viene, de alguna manera, reconocida por su inclusión sistemática en los catálogos de las llamadas empresas de Economía Social. Y aunque este concepto de Economía Social es difícil de concretar, ya que hace referencia una realidad muy diversa, generalmente, podemos entender como tal, y siguiendo a Vargas Vasserot "la actividad económica de carácter privado, basada en la asociación de personas en entidades de tipo democrático y participativo, con primacía de las aportaciones personales sobre las de capital, donde la distribución del beneficio y la toma de decisiones no están directamente ligadas con el capital aportado por cada socio" (Vargas Nasserot 2010: 78).

Independientemente del ordenamiento y configuración de las SAT, de tipo social, aún con defectos, funciona, tal y como muestra el caso de "la Casadel Arzipreste", en la que Claudia, alma mater de la experiencia, se ha manifestado una experta: Los que eligen esta fórmula han visto claras sus ventajas, sobre todo si se compara con las cooperativas. Las SAT no tienen ninguna de las típicas limitaciones de las sociedades cooperativas, generalmente derivadas de su peculiar estructura organizativa, límites financieros, límites operativos o funcionales, etc.

\section{WWOOF como desarrollo de la SAT}

WWOOF, siglas de willing workers on organic farms, o, como lo traducen en forma libre, ayudantes voluntarios en granjas y tareas rurales ecológicas, es una red internacional que pretende ser útil tanto a las granjas rurales que trabajan principalmente con métodos ecológicos y sostenibles, como a los voluntarios o wwoofers que quieren trabajar en ellas. En este sentido, sirve, de forma inmediata, para coordinar la oferta de granjas que aceptan ayuda voluntaria y la demanda de voluntarios o wwoofers que desean experimentar y ayudar en un entorno rural, o como dice el lema de la asociación, con marcado carácter anglófilo, World Wide Opportunities on Organic Farms: living, learning, sharing organic lifestyles.

La relación entre granjas y los wwoofers siempre se plantea de forma libre, voluntaria, sin existir ningún tipo de compensación económica ni de otros bienes. La red permite mantener un listado de granjas mediante el cual, todos aquellos que pretendan tener esta vivencia, y generalmente, para aprender o perfeccionarse, se pueden poner en contacto con la granja elegida y acuerdan los términos de la estancia. Este acuerdo puede producirse de forma individual o por grupos o por familias con hijos incluidos. Por tanto, sirve también de canal de comunicación, para facilitar y mediar entre unas y otros, siempre dentro del clima de voluntariado, en la legalidad vigente en cada país y en la estructura jurídica en que se encuentre la granja. Lo que se pretende, en definitiva, es facilitar conocimientos y experiencias 
sobre técnicas de agricultura ecológica de una manera práctica. También, organiza y difunde proyectos basados en el voluntariado, para desarrollar el interés y el conocimiento de las técnicas avanzadas en el cultivo ecológico, la defensa del medio ambiente y de la economía social. Todo ello, además, de cara a la sociedad en general, permite presentar la agricultura ecológica como una alternativa viable. De alguna forma, el objetivo general que persigue es doble, promover la solidaridad entre las personas y una economía ética, y dentro del ámbito del absoluto respeto por la naturaleza. Aunque la organización comparte fines comunes con las granjas, ambas trabajan por separado.

Pero ¿cómo se traduce esto para el caso que nos ocupa? "La Casadel Arzipreste" es una sociedad agraria de transformación que está integrada en esta red. Según nos dice Claudia, el alma mater de esta experiencia, "los wwoofers acogidos no tienen remuneración alguna, pero a cambio pueden disfrutar de la residencia en la casa rural, tienen comida sana, procedente de los huertos ecológicos, y sobre todo, se llevan los conocimientos para una vida más sostenible", que pueden comenzar en otro lugar o continuar en su lugar de origen. "Entre las exigencias antes del acuerdo, es imprescindible el interés genuino por la alternativa ecológica, por lo ecológico".La Granja se compone de varias fincas y monte y edificios. "Generalmente se necesita ayuda en la huerta, en el mantenimiento de plantaciones de árboles, en la restauración de edificios y tapias del pueblo, en la conservación de las cosas cosechadas y mucho más... Tenemos cuatro hectáreas con muchos árboles, madera, frutales, monte y pradería, huerta, jardín...".

La filosofía subyacente es la reproducción del sistema y así, en los casi tres años que llevan en Requejo, ya han pasado por la granja una treintena de personas, de muy diverso origen social y geográfico, con un carácter marcadamente internacional, integradas en este movimiento, "siempre con la idea de aprender, copiar el modelo y establece su propia granja en otro lugar". Y el planteamiento funciona, pues tenemos recordar que algunos de los hoy habitan Requejo habían estado anteriormente viviendo en Matavenero. Y ahora, "algunos de los que han pasado por "La Casadel Arzipreste" se han ido a fundar otra comunidad". Por ejemplo, aquí vive ahora Eldar, de 21 años y también de origen alemán. Junto con unos amigos tiene previsto establecer otra comunidad "en algún lugar mágico del sur de España". Dice: "el mundo está muy mal y si estás aislado, estás más seguro. Lo que tampoco puedes pensar es que esto es un oasis". Intentará llevar lo mejor de lo que aprendió aquí. Como wwoofers dentro de una SAT, se plantea "evolucionar en el concepto de grupo, promoviendo el trueque de productos entre aldeas". Y con el tiempo, criará a sus hijos: "les enseñaré que ensuciarse es bueno". Mientras tanto, lo que propone un modelo de sociedad ecológica con sistema de autogestión. En el imaginario social de este modelo de neoruralismo, se afirma un cuestionamiento de la sociedad industrial, por sus aspectos de consumo sin fin, de explotaciones abusivas de los recursos naturales, por su lado materialista y mercantil de la sociedad de consumo como fin en sí mismo. Trasluce una protesta contra el proceso de desertización masiva de los pueblos y una exaltación de la vida en comunidad de los pequeños pueblos, celebrando la grandeza de la vida rural. Y es la desaparición de ese mundo lo que, con el tiempo, genera nostalgia. Sucede algo similar a lo que sucedía en el período de 1950-60, cuando el abandono del campo estaba marcado por lo que H. Lefebvre llamó "el aumento de la nostalgia" (cit. por Nates Cruz y Raymond 2007), una suerte de movimiento caracterizado por la separación del "hombre y de la naturaleza". Por eso hoy, el neoruralismo se presenta al mismo tiempo como una mezcla de protesta social, de nostalgia con relación a un pasado rural realizado, de corte "naturalista" y de renovación. Y así, a la obsesión de un "pueblo abandonado", se ha respondido con un "pueblo reencontrado". Y es que, como argumenta Díaz Viana "la gente se va y regresa por algo; consigue hacerlo satisfactoriamente en mucho casos. (...) Volver a un lugar en el que nos se había estado antes es una evasión". Por eso, frente a esa nostalgia se propone una evasión. Una evasión en lo nos concierne más que nada, es "el territorio imaginado, culturalmente construido, más que el territorio físico, geográfico, tenido hasta hace poco como el único real. No se busca tanto la naturaleza como un tiempo perdido y añorado. No se busca tanto un espacio como una identidad" (Díaz Viana 2011: 92).

\section{Conclusiones}


Hemos expuesto un planteamiento de la neo-ruralidad como proceso inscrito en un movimiento amplio y global, que se inicia en el periodo de finales de los años 60 del siglo XX, que tiene su continuidad en la sociedad actual, y se asienta en un sistema de vida, consumo e ideología muy preciso en relación con la tierra y la naturaleza. Frente a las consideraciones peyorativas del campo en la época de abandono y despoblación rural, el creciente interés por esta nueva relación con el entorno rural desvela la desilusión de la vida urbana y el desencanto de los urbanitas. Los cambios en la estructura económica del campo, su urbanización, vaciado de funciones agrarias tradicionales ciertamente ponen en juego el proceso de gentrificación del medio rural.

Hemos visto la estrategia desarrollada para la integración de una experiencia de nueva ruralidad en el sistema económico y social, a través la casa rural denominada "La Casadel Arzipreste" que, con una configuración de economía social a través de una sociedad agraria de transformación, y de su integración en una red internacional de granjas orgánicas con voluntariado, ha demostrado que no hay zonas rurales sin futuro, sino zonas rurales sin proyectos.

Aunque cuantitativamente, como viene sucediendo en casos similares, no es un fenómeno importante, si es significativo considerando los cambios que producen, y no sólo en el ámbito socioeconómico. Evidentemente, los nuevos llegados son el origen de las iniciativas que permitirán suplir la falta de servicios en la comarca.

Los discursos suscitados en las historias personales de los neo-rurales participantes en la experiencia, muestran la huida de la urbe agobiante y tediosa, pero también un reencuentro con el entorno rural, imaginario o real, lo que desvela la oscilación que se produce entre la evasión de la urbe y la nostalgia por un campo reencontrado.

\section{Notas}

1. Este trabajo se encuadra dentro del Proyecto de investigación titulado "El retorno a la tierra. Problemas, dinámicas y estrategias de la neo-ruralidad", financiado por el Plan Nacional I+D+I del Ministerio de Ciencia e Innovación, referencia FFI2009-12186.

\section{Bibliografía}

Cervantes, R.

2008 "La Sociedad Agrariade Transformación en España: un análisis histórico", CIRIEC, nº 63: 65-87.

De Ravignan, F.

1996 "Des citadins en mal de champagne", Vivre Autrement, $\mathrm{n}^{0} 2$.

Delgado Campos, $\mathrm{J}$.

1999 "Nueva ruralidad en México", Investigaciones geográficas, n 39: 82-93.

Di Méo, G.

1998 Géographie sociales et territories. Paris, Nathan Université.

Díaz Viana, L.

2011 Viana de Cega: entre dos ríos. Etnografía, Historia y medio natural de un pueblo de Tierra de Pinares. Valladolid, Diputación de Valladolid.

Folch Monclús, R. 
2011 "El movimiento neorrural en el Prepirineo de Lleida: el caso de la Terreta", en L. Díaz, O. Fernández y P. Tomé (coords.), Lugares, Tiempos, Memorias. La antropología Ibérica en el siglo XXI. León, Universidad de León: 2197-2206.

Harper, S.

1987 "A humanistic approach to the study of rural population", Journal of Rural Studies, $n^{0} 3: 309-319$.

Lara, S.

1996 "El papel de las mujeres en la nueva estructura de los mercados de trabajo laborales rururbanos", en A. De Teresa y C. Cortez (coords.), La nueva relación campo-ciudad y la pobreza rural. México, INAH, Plaza y Valdés: $145-165$.

Long, N.

1996 "Globalización y localización: nuevos retos para la investigación rural", en S. Lara Flores y M. Chauvet (coords.), La inserción de la agricultura Mexicana en la economía mundial. México, Plaza y Valdés: 35-74.

Nates Cruz, B. (y S. Raymond)

2007 Buscando la naturaleza. Migración y dinámicas rurales contemporáneas. Barcelona, Anthropos.

Nates Cruz, B. (coord.)

2010 "Nuevos territorios e innovación digital virtualidad, diversidad cultural y construcción social de los espacios", Anthropos, nº 227 (monográfico).

Pereiro, X. (S. Prado Conde y S. Roseman)

2011 "Antropología y Nuevas ruralidades" mesa de trabajo presentada en el XII Congreso de Antropología de la FAAEE, Lugares, Tiempos, Memorias. La antropología Ibérica en el siglo XXI. León, Universidad de León: 2155-2236.

Rubio Pérez, L.

1993 El sistema político concejil en la provincia de León. León, Universidad de León.

Salas Quintanal, H. J. (M. L. Rivermar Pérez y P. Velasco Santos) (coords.)

2011 Nuevas Ruralidades. Expresiones de transformación social en México. Mexico, UNAM.

Smith, N.

1996 The New Urban Frontier: Gentrification and the Revanchist City. London, Routledge.

Vargas Vasserot, C.

2010 "Las sociedades agrarias de transformación en España. Defectos legales y ventajas operativas", Estudios agrarios, $\mathrm{n}^{\circ}$ 2: 75-107. 